\title{
Finite-Volume Scaling of the Quenched Chiral Condensate*
}

\author{
Poul H. Damgaard ${ }^{\mathrm{a}}$, Robert G. Edwards ${ }^{\mathrm{b}}$, Urs M. Heller ${ }^{\mathrm{b}}$, and Rajamani Narayanan ${ }^{\mathrm{c}}$ \\ a The Niels Bohr Institute, Blegdamsvej 17, DK-2100 Copenhagen, Denmark \\ b SCRI, The Florida State University, Tallahassee, FL 32306-4130, USA \\ c American Physical Society, One Research Road, Ridge, NY 11961, USA
}

In the large-volume limit $V \rightarrow \infty$ with $V \ll 1 / m_{\pi}^{4}$ the mass-dependent chiral condensate is predicted to satisfy exact finite-volume scaling laws that fall into three major universality classes. We test these analytical predictions with staggered fermions and overlap fermions in gauge field sectors of fixed topological charge $\nu$.

\section{Finite-volume partion functions and RMT}

Surprisingly, there exists a deep connection between finite-volume gauge theories with spontaneous breaking of chiral symmetries, and Random Matrix Theory (RMT). In the chiral limit $m_{i} \rightarrow 0$ such that $\mu_{i} \equiv m_{i} \Sigma V$ are kept fixed, the field-theoretic partition functions $Z_{\nu}\left(\left\{\mu_{i}\right\}\right)$ become identical to certain (chiral) RMT partition functions [1]. Here $\Sigma$ denotes the (conventional) infinite-volume chiral condensate, and $\nu$ is the (fixed) topological charge of the gauge fields. This remarkable identity of partition functions is stable under huge perturbations of the RMT “potential”, i.e. universal [2]. RMT plays a role here because the three major (chiral) classes of RMT ensembles correspond to the cosets of spontaneous chiral symmetry breaking. It is crucial that one considers the limit $V \rightarrow \infty$ with $V \ll 1 / m_{\pi}^{4}$. Taken conversely, from reading off the scaling behavior of the finite-volume chiral condensate one deduces the coset of spontaneous chiral symmetry breaking!

From the universal relationship between these different expressions for the finite-volume partition functions follows that there are also universal scaling relations for the chiral condensate and higher chiral susceptibilities. Because the pertinent chiral RMT ensembles are presumed relevant also for systems in condensed matter physics,

\footnotetext{
*Presented by P. H. Damgaard
}

these universal scaling laws indeed go much beyond their immediate context of four-dimensional Yang-Mills theories.

The quenched limit can be taken in both the RMT framework [3] and that of the effective lagrangian [4]. The RMT framework provides a neat classification of the universality classes, and we shall employ this RMT language below. We work in the quenched limit.

\section{Staggered Fermions}

At moderate gauge couplings staggered fermions are almost blind to topology [5] (but see also ref. [6]), and we thus should compare analytical predictions to those of $\nu=0$ only. We have performed a systematic analysis of all three different universality classes in ref. [7] . The first study of this kind was performed by Verbaarschot [3] for the chUE universality class.

There are exact analytical predictions for all three universality classes [7]. We here concentrate on just one of these, the chOE. For staggered fermions away from the continuum limit it corresponds to all gauge groups $\mathrm{SU}\left(N_{c} \geq 2\right)$ and fermions in the adjoint representation. The quenched chiral condensate for that case has the analytical form (for $\nu=0$ ):

$$
\begin{array}{r}
\frac{\Sigma_{0}^{\operatorname{chOE}}(\mu)}{\Sigma}=\mu I_{1}(\mu) K_{1}(\mu)+\frac{\pi}{2}\left[I_{0}(\mu)-\mathbf{L}_{0}(\mu)\right] \\
+\frac{\pi}{2} K_{0}(\mu)\left(\mathbf{L}_{0}(\mu) I_{1}(\mu)-\mathbf{L}_{1}(\mu) I_{0}(\mu)\right] .
\end{array}
$$

Eq. (11) gives a parameter-free prediction for the 


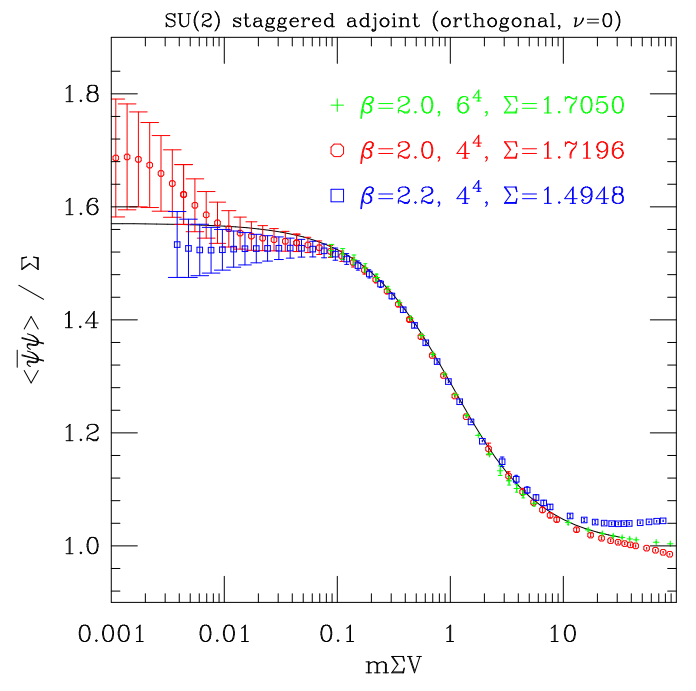

Figure 1. The rescaled quenched consensate for staggered fermions in the the adjoint representation of $\mathrm{SU}(2)$.

scaling of $\Sigma_{0}(\mu)$ once $\Sigma$ is known. We compare this prediction with lattice data in Fig. 1. Note the unusual behavior: The condensate rises with decreasing mass, eventually saturating at the constant value $\Sigma_{0}(\mu) / \Sigma=\pi / 2$. Here, as $V$ is taken to infinity, the mass $m$ is taken to zero at a rate proportional to $1 / V$. This would normally imply that $\Sigma(\mu) \rightarrow 0$ as $\mu \rightarrow 0$. The fact that it doesn't here is due to the quenched approximation, and the $\nu=0$ sector.

We have compared the analytical predictions for all three universality classes using staggered fermions, and found remarkably good agreement, even in rather small physical volumes [7]. One sees very clearly the striking scaling of the condensate: instead of depending on the three parameters $m, \Sigma$ and $V$, it only depends on the scaling combination $\mu=m \Sigma V$.

\section{Topology: Overlap Fermions}

It is far more interesting to test the analytical predictions with lattice fermions that are sensitive to lattice gauge field topology. Results have previously focused on the smallest eigenvalue dis- tributions [8]. We shall here present some of our results for the chiral condensate, obtained using overlap fermions [9]. Similar results for the case of the chUE universality class have been presented in ref. [10]. In contrast to staggered fermions, overlap fermions have the additional advantage of belonging, at finite lattice spacing, to precisely the same universality classes as continuum fermions.

The massive overlap Dirac opertor is given by

$D(m)=\frac{1}{2}\left[1+m+(1-m) \gamma_{5} \epsilon\left(H_{w}(m)\right)\right]$.

and the propagator for external fermions needs a subtraction to ensure the proper chiral behavior in the massless limit,

$\tilde{D}^{-1}(m)=(1-m)^{-1}\left[D^{-1}(m)-1\right]$.

The condensate $\Sigma(m)=\left\langle\operatorname{Tr} \tilde{D}^{-1}(m)\right\rangle$ can then be easily obtained using Gaussian random source methods [11].

Overlap fermions have exact zero modes in topologically non-trivial gauge fields [8], and allow therefore comparison with RMT predicitions in sectors with $\nu \neq 0$. We separate out the rather trivial contribution from the exact zero modes and consider $\Sigma_{\nu}(\mu) / \Sigma-|\nu| / \mu$. For its stochastic estimate, we use the fact that $D^{\dagger}(m) D(m)$ commutes with $\gamma_{5}$, from which follows that the contribution from the non-zero modes to $\Sigma_{\nu}$ is equal in both chirality sectors so that we can choose to do the necessary inversion only in the sector without zero mode 11]. Finally, using

$D^{\dagger}(m) D(m)=\left(1-m^{2}\right)\left[D^{\dagger}(0) D(0)+\frac{m^{2}}{1-m^{2}}\right](4)$

we can simultaneously compute for several masses using multiple Krylov space solvers.

We considered examples for all three ensembles of the RMT classification [7]. Our results are shown in Fig. 2. In all cases the condensate $\Sigma$ had been determined previously from the distribution of the lowest non-zero eigenvalue [8] and the RMT predicitions, shown as curves in Fig. 目, are therefore parameter free.

Due to the costs of the simulations with overlap fermions, we were restricted to rather small volumes and relatively low statistics. As discussed 

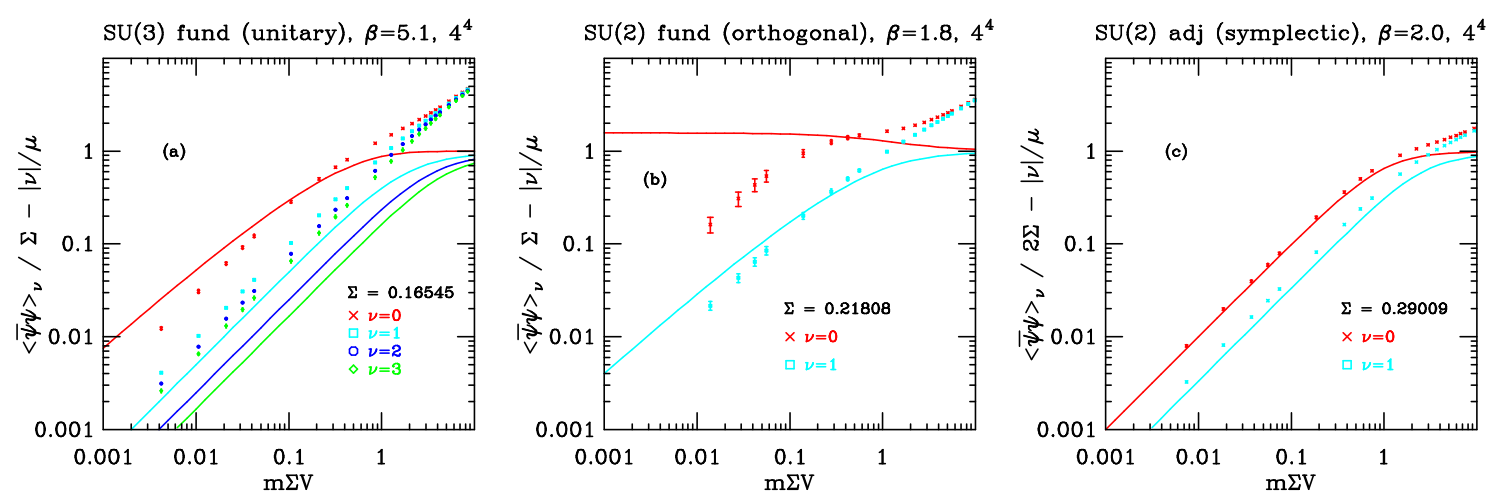

Figure 2. The rescaled quenched condensate as a function of $\mu=m \Sigma V$ for overlap fermions in (a) the fundamental representation of $\mathrm{SU}(3)$, (b) the fundamental representation of $\mathrm{SU}(2)$, (c) in the adjoint representation of $\mathrm{SU}(2)$.

in detail in [7], the deviations seen in Fig. 2 from the analytical predicitions are readily understood if one considers the microscopic spectrum of the Dirac operator. One is effectively caught between the difficulty of probing the very smallest eigenvalue to very high accuracy, and the finite-volume limitation that distorts the microscopic Dirac operator spectrum after just a few (averaged) eigenvalue peaks. The best agreement with analytical predictions are found in the case of the chSE universality class, in both the sector of $\nu=0$ and $\nu=1$. This is again a reflection of the microscopic Dirac operator spectrum for those gauge field configurations [7]. Even for the other two universality classes the results are qualitatively in good agreement with theory, in particular the shift due to higher topological charge sectors is correctly reproduced.

The work of R.G.E. and U.M.H. was partially supported by DOE contracts DE-FG0585ER250000 and DE-FG05-96ER40979, and the work of P.H.D. by EU TMR grant ERBFMRXCT97-0122. Both P.H.D. and U.M.H. also cknowledge support by NATO Science Collaborative Research Grant CRG 971487. All the authors acknowledge the hospitality of the Aspen Center for Physics, were this research was completed.

\section{REFERENCES}

1. E.V. Shuryak and J.J.M. Verbaarschot, Nucl. Phys. $A 560$ (1993) 306; M.A. Halasz and J.J.M. Verbaarschot, Phys. Rev. D52 (1995) 2563; J.J.M. Verbaarschot, Phys. Rev. Lett. 72 (1994) 2531.

2. G. Akemann, P.H. Damgaard, U. Magnea and S. Nishigaki, Nucl. Phys. B487 (1997) 721.

3. J.J.M. Verbaarschot, Phys. Lett. $\mathbf{B 3 6 8}$ (1996) 137.

4. J. Osborn, D. Toublan and J.J.M. Verbaarschot, Nucl. Phys. B540 (1998) 317; P.H. Damgaard, J. Osborn, D. Toublan and J.J.M. Verbaarschot, Nucl. Phys. B547 (1999) 305.

5. P.H. Damgaard, U.M. Heller, R. Niclasen and K. Rummukainen, hep-lat/9907019.

6. F. Farchioni, I. Hip and C.B. Lang, heplat/9907011.

7. P.H. Damgaard, R.G. Edwards, U.M. Heller and R. Narayanan, hep-lat/9907016.

8. R.G. Edwards, U.M. Heller, J. Kiskis and R. Narayanan, Phys. Rev. Lett. 82 (1999) 4188; F. Farchioni, I. Hip, C.B. Lang and M. Wohlgenannt, Nucl. Phys. B549 (1999) 364.

9. H. Neuberger, Phys. Lett. B417 (1998) 141.

10. P. Hernandez, K. Jansen and L. Lellouch, hep-lat/9907022.

11. R.G. Edwards, U.M. Heller and R. Narayanan, Phys. Rev. D59 (1999) 094510. 